
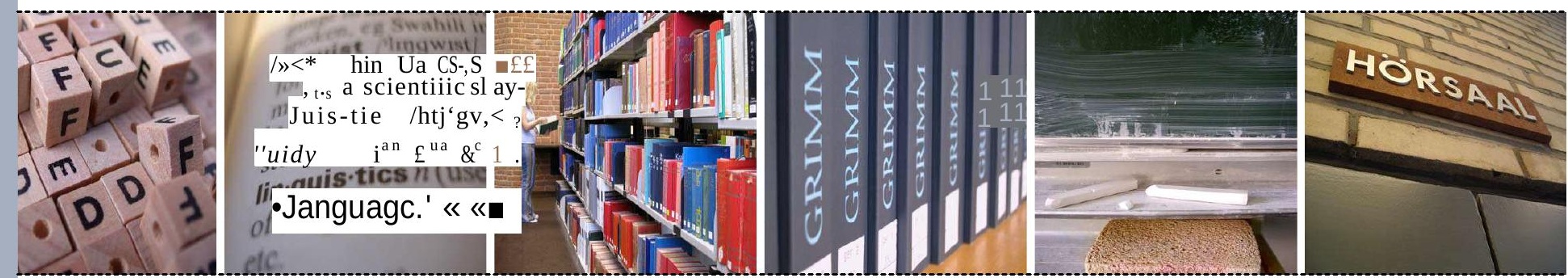

\author{
RUDI KELLER
}

\title{
IST DIE DEUTSCHE SPRACHE VOM VERFALL BEDROHT?
}




\section{INHALTSVERZEICHNIS}

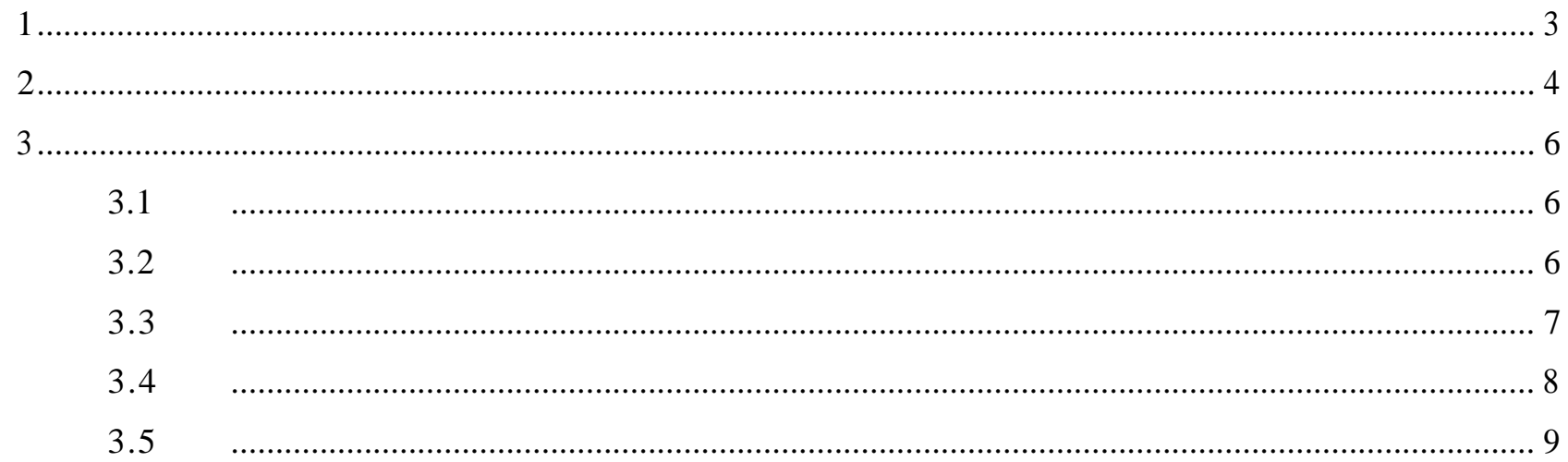


1.

Jede Zeit sagt, daß derzeit die Sprache so gefährdet und von Zersetzung bedroht sei wie nie zuvor. In unserer Zeit aber ist die Sprache tatsächlich so gefährdet und von Zersetzung bedroht wie nie zuvor. [...] Der Journalismus ist schuld, der geschriebene Journalismus und der gesprochene des Radios und des Fernsehens. [...] Die Bildungsexplosion hat beträchtlichen Sprachschaden angerichtet. Und das Schrecklichste an der Bildungsexplosion ist nicht die ständig wachsende Zahl von Schülern und Studenten, sondern die ständig wachsende Zahl von Lehrern und Professoren. ${ }^{1}$

Die Sprache wird heute so schnell umgebildet, daß sie heute verkommen und verlottert ist. Unbeholfenheit und Schwerfälligkeit, Schwulst, Ziererei und grammatische Fehlerhaftigkeit nehmen zu. An die Stelle einer guten Schriftsprache ist eine häßliche Papiersprache getreten. Dazu kommt noch die Ausländerei, eine Erbschwäche des Deutschen. Der Deutsche mag so alt werden, wie er will, er wird immer und ewig der Affe der anderen Nationen bleiben. Franzosennachäfferei und Engländernachäfferei sind verbreitet. [...] Der eigentliche Herd und die Brutstätte dieser Verwilderung sind die Zeitungen, genauer die Tagespresse. Seit der Pressefreiheit von 1848 gibt es ein Überangebot, das zur Verwilderung führt. [...] Vor allem sind die Juden an diesem Verfall schuld: Ein großer Teil unseres heutigen Sprachunrats geht ausschließlich auf das Judendeutsch der Berliner und Wiener Tagespresse zurück. Der Grund dafür ist, daß die Vorfahren der Juden noch nicht Deutsch als Muttersprache sprachen. Deshalb beherrscht der Jude die deutsche Sprache nicht so gut: So flink sich auch der Jude in die Elemente der deutschen Grammatik findet, wo es aufs Sprachgefühl ankommt, bleibt er doch ewig der Fremde. [...] Die eigentlich Schuldigen sind aber in der Schule zu suchen: Wo stammen sie denn her, die Deutschverderber der letzten vierzig Jahre, wenn nicht aus der deutschen Schule? Wir haben ja gar keinen deutschen Unterricht! ${ }^{2}$

Diese beiden Klagen liegen knapp hundert Jahre auseinander, aber in ihrem Wesen sind sie sich abgesehen von Wustmanns rassistischen Motiven - ziemlich ähnlich: Die deutsche Sprache ist in akuter Gefahr, sie verludert; und schuld daran ist die Flut der »neuen« Medien und die Schule. Das ist der Topos des Sprachverfalls. Ich denke, jeder von uns kam mit dieser linguistischen Variante des Kulturpessimismus' irgendwann einmal in Berührung. Und wenn man Wustmanns Klage reinigt von den »ethnolinguistischen« Ergüssen über die »Schuld des Juden« im Prozess des Sprachverfalls, dann könnte so mancher geneigt sein, im Stillen zu denken: »Ja, im Grunde genommen hat er recht.« Immerhin hat Wustmanns Buch bis 1966 vierzehn Auflagen erlebt, allerdings ohne das Vorwort der ersten Auflage. Der Topos des Sprachverfalls ist allgegenwärtig - in allen Kulturnationen und über alle Zeiten hinweg: von Platon über Quintilian und Rousseau bis hin zu Kemal Pascha, Helmut Kohl oder Prinz Charles, der unlängst seine Sorge artikulierte über die zunehmende Verunreinigung der britischen Variante des Englischen durch die amerikanische. Esvergeht kaum eine Woche, in der nicht

1 Hans Weigel: Die Leiden derjungen Wörter, Munich 1974.

2 Gustav Wustmann: Allerhand Sprachdummheiten. Kleine deutsche Grammatik des Zweifelhaften, des Falschenund des Hässlichen, Leipzig 1891. 
ein Leser der Rheinischen Post in einem Leserbrief seine Ängste über die Zukunft der deutschen Sprache zum Ausdruck bringt.

Sind diese Sorgen berechtigt? Und woher rühren sie? Diesen und verwandten Fragen soll dieser Beitrag gewidmet sein.

2.

Zunächst einmal fällt dreierlei auf:

1. Seit mehr als zweitausend Jahren ist die Klage über den Verfall der jeweiligen Sprachen literarisch dokumentiert, aber es hat bislang noch nie jemand ein Beispiel einer »verfallenen Sprache« benennen können.

2. Vom Verfall bedroht ist offenbar immer die jeweils zeitgenössische Version der jeweiligen Sprache. Kein britischer Prinz würde beispielsweise heute darüber klagen, dass das wundervolle Angelsächsisch zu dem völlig gallifizierten Neuenglisch verkommen ist.

3. Sprachkritik ist - und auch das sollte zu denken geben - stets Fremdkritik, Kritik am Sprachgebrauch der anderen. Die Klage »Was schreibe ich doch für ein verwahrlostes Deutsch im Vergleich zu meinen Großeltern«, diese Form der Selbstkritik ist äußerst rar.

Das vorherrschende Bild ist folgendes: Der gegenwärtige Zustand meiner Sprache ist der korrekte, gute und schöne, und von nun an geht's rapide bergab. Im 19. Jahrhundert wurde dieses Bild des Sprachverfalls oft noch theoretisch untermauert durch die Organismus-metapher: Die Sprache ist ein Organismus, und bei Organismen ist war es schon immer so, dass sie »nach bestimmten Gesetzen wuchsen und sich entwickelten und wiederum altern und absterben «. ${ }^{3}$ Diesem Organismus-Denken ist es wohl auch zu verdanken, dass wir eine Sprache wie das Latein als »tote« Sprache bezeichnen. In Wahrheit ist Latein natürlich nicht tot, sondern lebt munter weiter in Formen, die man gemeinhin »französisch«, »italienisch«, »katalanisch« usw. nennt; so gesehen ist französisch nichts anderes als verfallenes Latein: Die schöne klassische Form cantabo »ich werde singen« wurde im Vulgärlatein ersetzt durch die analytische Umschreibung cantare habeo und diese dann - durch die schludrige Aussprache der Gallier - sukzessive über cantare aio zu der französischen Form chanterai heruntergewirtschaftet. Und damit nicht genug: chanterai wird mehr und mehr verdrängt durch eine neue vulgäre Umschreibung je vais chanter. So etwa müsste eine sprachgeschichtliche Beschreibung aussehen, wenn man die Sprachentwicklung mit den Augen eines Verfallstheoretikers betrachten würde.

Und damit bin ich bei meiner zentralen These: Was wir als Sprachverfall wahrnehmen ist der allgegenwärtige Sprachwandel, aus der historischen Froschperspektive betrachtet. Wir beobachten die

3 August Schleicher 1863. 
Sprache punktuell durch ein schmales Zeitfenster und erkennen in diesem begrenzten Ausschnitt notwendigerweise jede Menge Fehler und Barbarismen. Die systematischen Fehler von heute sind jedoch mit hoher Wahrscheinlichkeit die neuen Regeln von morgen. Ich will versuchen, dies an einer Reihe von Beispielen zu belegen und zu veranschaulichen.

Es ist wichtig, vorab zu klären, in welchem Sinne man das Wort Sprache verwendet, wenn man über deren Verfall redet; denn dieses Wort wird in unserer Umgangssprache äußerst vieldeutig gebraucht. In den Ausdrücken »die Sprache des jungen Goethe«, »die Sprache der Jugendlichen« und »die deutsche Sprache« wird »Sprache« in je verschiedener Bedeutung verwendet. Im ersten Fall meint man einen bestimmten Ideolekt, eben die typischen Besonderheiten des Sprachgebrauchs des jungen Goethe; im zweiten Fall eine bestimmte gruppenspezifische Varietät des Deutschen, einen bestimmten Soziolekt. Sprache im Sinne von Deutsch, Englisch oder Suaheli meint ein bestimmtes System von konventionellen Regeln - phonologischen, syntaktischen und semantischen Konventionen - die gegenwärtig gelten. Da gesellschaftliche Konventionen ständigem Wandel unterliegen und außerdem (beispielsweise) sozial, regional, alters- und möglicherweise geschlechtsspezifisch variieren, ist der Begriff »die deutsche Sprache« notwendigerweise äußerst unscharf. Aber immerhin gibt es einen harten Kern derselben, sagen wir die Schnittmenge all dieser Varietäten. In einem solchen Sinne ist »Sprache« wohl gemeint, wenn vom Verfall derselben die Rede ist. Damit ist aber auch klar, dass man Sprache nicht gleichsetzen darf mit bestimmten Äußerungen oder Texten einzelner Personen. Fehlerhafte Äußerungen lassen keine Schlüsse auf den Zustand »der Sprache« zu - es sei denn, es handelt sich um sehr systematisch und frequent vorkommende Fehler. Damit sind wir an einem Punkt, der ausschlaggebend sein könnte für die verbreitete Ansicht, die Sprache gehe allmählich zugrunde: Jede Veränderung einer Konvention beginnt notwendigerweise mit deren Übertretung, und Übertretungen sprachlicher Konventionen nennt man »Fehler«. Wenn der Fehler schließlich zum allgemeinen Usus geworden ist, dann hat er aufgehört, ein Fehler zu sein und eine neue Konvention ist entstanden. Solange das Präteritum des Verbs schrauben noch schrob lautete, machte der, der schraubte sagte, einen Fehler. Heute machen wir alle diesen »Fehler« und genau deshalb ist es keiner mehr. (Erhalten geblieben ist uns nur noch die starke Form des Partizips von verschrauben in seiner metaphorischen Bedeutung: verschroben.) Dieses Beispiel macht deutlich, weshalb aufmerksame Sprachbeobachter immer und überall den Eindruck gewinnen müssen, dass ihre Sprache verwahrlost. Wir nehmen den Beginn eines Wandelsprozesses wahr, der notwendigerweise eine Regelverletzung darstellt. Unsere Wahrnehmung lässt nach in dem Maße, in dem die anfängliche Regelverletzung zum allgemeinen Usus wird. Denn damit verliert sie jede Auffälligkeit. Sehr deutlich wird dies an orthografischen Regeln: Wer heute vorschlägt, Spaghetti ohne »h« zu schreiben, riskiert den Vorwurf, 
die abendländische Kultur zu schänden (wobei er den Plural Spaghettis vermutlich ungestraft bilden darf). Wer aber das englische Wort cWresals Keks schreibt und dies (wie bei Straps) auch noch für eine Singularform hält, macht sich keines Frevels verdächtig. Der Grund für die allgemeine Toleranz den Keksen gegenüber liegt aber ausschließlich darin, dass diese Schreibweise bereits vor 90 Jahren eingeführt wurde und wir uns mittlerweile daran gewöhnt haben.

3.

Welche Phänomene sind es, an denen der Verdacht des Sprachverfalls festgemacht wird? Im Zentrum der Kritik stehen gemeinhin grammatische Fehler, der Gebrauch von Fremdwörtern und neuerdings natürlich auch die Orthografie. ${ }^{4}$ Betrachten wir einige Beispiele aus diesen drei Bereichen. Beginnen will ich mit einem verbreiteten Argument gegen die neue Orthografie (gegen die man, nebenbei bemerkt, im Detail einiges vorbringen kann).

3.1

Sie wird dazu führen, so ist häufig zu hören, dass die Deutschen demnächst ihre eigenen Klassiker nicht mehr lesen können. Ein Blick in die Originalausgabe von Goethes Die Leiden des jungen Werthers von 1774 genügt, um die Absurdität dieser These zu belegen: »am 10. May ... Wenn das liebe Thal um mich dampft ... und näher an der Erde tausend mannigfaltige Gräsgen mir merkwürdig werden. Wenn ich ... die Gestalten all der Würmgen, der Mückgen ... das Wehen der Allliebenden ... Die Kühle des Orts, das hat so was anzügliches, was schauerliches. ... Ich bin ... in Verzükkung ... Ich saß ganz in mahlerische Empfindungen vertieft. ... um weis Brod zu holen, und Zukker. ... theils wegen dem Gegensazze«. Die Beispiele ließen sich beliebig fortsetzen. Sie zeigen nur, dass die Vertreter der oben genannten These die Texte unsere Klassiker noch nie in Originalorthografie gesehen haben. Ein Kind, das heute so schreiben würde, wie Goethe geschrieben hat, stünde in Deutsch glatt fünf.

\section{2}

Betrachten wir nun drei Beispiele systematischer grammatischer Fehler, die heute zu beobachten sind: im Herbst diesen Jahres werden wir umziehen. Sätze wie diesen hört man derzeit sehr oft, auch von gebildeten Sprechern. Natürlich sollte es im Herbst dieses Jahres heißen. Warum machen die Leute

4 »Die Rechtschreibreform ist belanglos angesichts der Verhunzung unserer Sprache durch die Ausbreitung des unsäglichen deutsch-englischen Sprachbreis«, Leserbrief im Spiegel vom 17.04.2000. 
genau diesen Fehler? Niemand käme schließlich auf die Idee die Hosen diesen Kindes oder der Fahrer diesen Autos zu sagen. Die Erklärung lautet: Eshandelt sich um eine Analogiebildung, ein Phänomen, das in der Entwicklung einer Sprache sehr häufig passiert. Es heißt im Herbst letzten, vorigen, nächsten Jahres, also auch im Herbst diesen Jahres. Die semantische Reihenbildung von letzten, vorigen und nächsten verleitet die Sprecher offenbar dazu, das Demonstrativpronomen diesesINle das Adjektiv letztes zu flektieren. Ich habe es bereits angedeutet: Die systematischen Fehler von heute sind die neuen Regeln von morgen. Die folgende Analogiebildung geschah offenbar bereits gestern: Der Genitiv des femininen Substantivs die Nacht heißt bekanntlich der Nacht, auf keinen Fall jedoch des Nachts. Dennoch hat sich des Nachts eingebürgert analog zu den semantisch ähnlichen Ausdrücke des Morgens, des Mittags und des Abends, die allesamt Maskulina sind. Hier ist die Analogieform mittlerweile voll akzeptiert, im Falle von diesen Jahres befindet sich die Sprachkonvention gerade im Umbruch.

3.3

Unser zweiter Fall systematischer Regelabweichung hat sogar zu einer Bürgerinitiative unter dem Kampfruf »Rettet den Kausalsatz« geführt: Ich muss gehen, weil die Geschäfte machen gleich zu. Auch dieser »Fehler« ist derzeit weit verbreitet; und auch hier ist zu erwarten, dass er zur akzeptierten Norm werden wird. Welcher Prozess ist hier im Gange? Die Konjunktion n/e/7ist eine subordinierende Konjunktion und sollte deshalb mit Nebensatz-Wortstellung konstruiert werden. Nicht weil die Geschäfte machen gleich zu, sondern weil die Geschäfte gleich zumachen. Ein Blick auf größere historische Zusammenhänge zeigt, dass das Wörtchen n/e/7eine lange und bewegte Geschichte hinter sich hat: Im Mittelhochdeutschen war das Wort wiie noch ein Substantiv, das etwa "Zeitdauer« bedeutete. Daraus entwickelte sich eine Konjunktion weil mit zunächst temporaler Bedeutung. Heirate, weil du jung bist konnte noch Schiller schreiben und damit meinen ,während du jung bist'. Die temporale Bedeutung entwickelte sich schließlich zur kausalen Bedeutung, ein Prozess der in vielen Sprachen immer wieder zu beobachten ist, denn die kausale Interpretation einer temporalen Aussage ist oft naheliegend (vgl. engl. since). Die kausale Konjunktion well ist gegenwärtig im Begriff, zu einer so genannten epistemischen Konjunktion zu werden. Etwas verkürzt lässt sich das so verdeutlichen: Der »neue« n/e/Z-Satzantwortet nicht mehr auf die kausale Frage »Warum ist das so?«, sondern auf die Frage »Woher weißt Du das?« Deshalb ist beispielsweise folgender Dialog durchaus sinnvoll:

Ist Peter noch hier? - Nein, der ist schon weg, weil sein Auto steht nicht mehr im Hof. 
Der entsprechende Satz mit »korrekter« Nebensatzwortstellung hingegen wäre nicht ohne weiteres sinnvoll und dürfte deshalb wohl auch kaum vorkommen:

Ist Peter noch hier? - Nein, der ist schon weg, weil sein Auto nicht mehr im Hof steht.

Das Fazit lautet: Auch hier handelt es sich nicht um einen Prozess der Verwahrlosung der Sprache, sondern um eine langfristige Entwicklung eines temporalen Substantivs zu einer temporalen Konjunktion, die schließlich vorübergehend kausale Bedeutung annimmt und nun im Begriff ist, zu einer epistemischen Konjunktion zu werden.

\section{4}

Als letzten Fall einer morphologischen Neuerung will ich ein Beispiel betrachten, das wohl den meisten ziemlich barbarisch vorkommen dürfte, da der Prozess noch sehr am Anfang steht. Es gibt ein neues Wort, das Wort son, in seiner femininen Form sone. Wir verwenden es in Sätzen wie Son Ding hab ich noch nie gesehen. Der Dativ davon heißt übrigens som und soner : Mit som Ding/soner Maschine geht das wunderbar. Nun werden Sie vielleicht einwenden: Das ist kein neues Wort, sondern einfach eine Zusammenziehung von so ein, so wie er hat's eine Zusammenziehung von er hat es ist. Die Antwort darauf lautet: Es war einmal eine Zusammenziehung von so ein. Diese hat sich aber mittlerweile verselbstständigt; sie wird bereits als eigenständiges Wort empfunden. Historisch kommt so etwas häufig vor. Auch das Wort Adler begann als Zusammenziehung von adel are (»edler Adler«); aber mittlerweile erkennen wir das nicht mehr, was daran zu merken ist, dass wir der edle Adler nicht als inhaltliche Verdoppelung empfinden. Dass sich eine solche Zusammenziehung verselbstständigt hat, erkennt man daran, dass sie sich nicht mehr ohne Weiteres wieder auseinanderziehen lässt. Wir könnten in jedem beliebigen Kontext anstelle von /W'sauch hat e>5verwenden. Statt son können wir nicht in jedem Falle so ein sagen: ich habe ein son Ding gehabt, und jetzt ist es weg. Die Ausdrücke ein son und kein son lassen sich nicht zurückführen auf ein so ein bzw. kein so ein. Sie sind nur möglich, weil son bereits als eigenständiges Wort empfunden wird. Um was für ein Wort handelt es sich dabei? Um einen unbestimmten Demonstrativartikel. Bis son/sone in die Schriftsprache Eingang gefunden haben wird, dürften noch ein paar Jahrzehnte ins Land gehen. Aber wenn das passiert, so wird es sich hierbei nicht um einen Verfall handeln, sondern im Gegenteil um eine Komplettierung unseres Sprachsystems. Beim bestimmten Artikel verfügen wir über die Opposition zwischen Verund dieser. Beim unbestimmten Artikel ein gibt es derzeit keine analoge Opposition. 
In der gesprochenen Umgangssprache machen wir einen feinen Unterscheid zwischen ein bzw. ' $n$ und son, wie Sätze der folgenden Art zeigen, in denen die beiden Formen nicht ohne weiteres austauschbar sind: Da vorne an der Ecke ist son Metzger, der hat 'n unheimlich guten Mittagstisch. Das Althochdeutsch war, wie das Latein, eine artikellose Sprache. Die Artikel der germanischen und der romanischen Sprachen haben sich mit der Zeit aus ehemaligen Demonstrativpronomina entwickelt und neue Demonstrativpronomina sind entstanden. Esspricht einiges dafür, dass wir derzeit im Begriff sind, ein indefinites Demonstrativum herauszubilden, und damit die Lücke im System zu schließen:

der -» dieser

'n $\rightarrow$ son

\section{5}

Kommen wir nun zu unserem letzten Punkt, den Fremdwörtern. Unsere Sprache droht ihre Eigenständigkeit zu verlieren angesichts der »Ausbreitung des unsäglichen deutsch-englischen Sprachbreis«, wie der zitierte Spiegelleser drastisch formuliert. Ist die Befürchtung berechtigt?

In der Tat ist es so! Wir werden geradezu überflutet von englischen und pseudo-englischen (Handy, Talkmaster, usw.) Ausdrücken und gewinnen bisweilen den Eindruck, dass es täglich mehr werden. Das mag uns daran erinnern: Eine Sprache dient uns nicht nur zum Kommunizieren, sie dient auch zum Imponieren. Dieser Tatsache ist wohl die inflationäre Innovationsgeschwindigkeit im Jargon der Jugendlichen und der Werbung zu verdanken, aber auch, mindestens teilweise, die Flut des Gebrauchs englischsprachiger Ausdrücke. Imponierverhalten ist ein integraler Bestandteil menschlichen Verhaltensrepertoires, und der Gebrauch fremdsprachlicher Ausdrücke zur Dokumentation der eigenen Vorzüglichkeit hat eine lange Tradition. Nur die Quellensprache ändert sich von Zeit zu Zeit; das Französische eignete sich im 18. Jahrhundert hervorragend, gesellschaftlichen Rang zu simulieren und wurde schließlich karikiert im so genannten Dienstmädchenfranzösisch. Das Küchenlatein war die Karikatur der Dokumentation akademischer Bildung; und jetzt ist Englisch an der Reihe, von asset management und abgespaced über cash flow und cool bis zu zero bonds und zappen. Einiges davon ist durchaus pfiffig, vieles überflüssig und das Meiste davon ist schlicht peinlich. Aber auch hier können wir die Frage stellen: Leidet das System der deutschen Sprache darunter? Und auch hier bin ich der Meinung, dass die Antwort negativ ausfällt. Ich will nur drei Gründe für diese Ansicht vortragen: 
1. Nicht jede ad-hoc-Entlehnung findet Eingang in die Sprache. Die meisten bleiben Eintagsfliegen oder saisonale Modeerscheinungen. Für unsere Enkelkinder werden die Ausdrücke cool und grooving so attraktiv sein wie für uns heute knorke oder dufte. Das Wort Handy wird uns möglicherweise erhalten bleiben und - wer weiß - irgendwann einmal in adaptierter Orthografie als Händi ein so mausgraues Dasein führen wie heute die Anglizismen Streik, Keks, Gully oder Tank. Und dass Handy eigentlich nicht englisch ist, wird uns so wenig stören wie die Tatsache, dass Plantage alA französisch eigentlich plantation heißt.

2. Ein Blick in das Orthographische Wörterbuch der deutschen Sprache, das Konrad Duden 1880 vorlegte, zeigt Folgendes: Allein unter dem Buchstaben »A« sind hunderte von Fremdwörtern verzeichnet, die im heutigen Duden nicht mehr aufgeführt sind, weil sie entweder völlig ungebräuchlich oder gar unbekannt sind: abalinleren, Abandonnement, abducieren, abhorrescieren, abhorrieren, abjurieren, ablaktieren, affrontieren, ambieren, Aneurie, attrapieren, azotisch, usw. Wer die Überfremdung befürchtet, sollte nicht nur die Zugänge in den Blick nehmen, sondern sie mit den Abgängen bilanzieren. Das hat meines Wissens noch niemand getan.

3. Was würde passieren, wenn tatsächlich einmal ein erheblicher Prozentsatz des Wortschatzes der deutschen Sprache englischen Ursprungs wäre? Das können wir an den Sprachen ablesen, bei denen ein analoger Prozess tatsächlich stattgefunden hat. Ein Musterbeispiel für eine »überfremdeten « Sprache ist das Englische: Etwa 30 bis 40 Prozent des englischen Wortschatzes sind französischen Ursprungs, dank der Normannischen Eroberung und der Tatsache, dass englische Adlige bis ins $18 \mathrm{Jh}$. hinein untereinander auf Französisch zu konversieren pflegte. Wie man sieht, hat dies der Attraktivität dieser Sprache und ihrer Tauglichkeit keinen Abbruch getan - ebenso wenig wie der überwältigende arabische Einfluss auf das Spanische oder der chinesische Einfluss auf das Koreanische.

Auch in Bezug auf die vermeintliche Bedrohung des deutschen durch fremde Elemente können wir feststellen: Sie ist geringer als vermutet; und selbst wenn sie erheblich wären, würde dies der Sprache auf lange Sicht weder funktional noch ästhetisch Schaden zufügen. Sprachzustände sind keine Endzustände von Prozessen, sondern flüchtige Episoden in einem potentiell unendlichen Prozess kultureller Evolution. Zu Sorge besteht kein Anlass - oder, wie Professor Rainer Wimmer, der ehemalige Direktor des Instituts für Deutsche Sprache, es einmal ausdrückte: »Die deutsche Sprache ist gut in Schuss.« 


\section{KONTAKT}

\section{Prof. Dr. Rudi Keller}

Heinrich-Heine-Universität Düsseldorf

Germanistisches Seminar

SI ANSCHRIFT

Universitätsstr. 1

40225 Düsseldorf

Si TELEFON:

+49 (0)211 81-12945

SI TELEFAX:

+49 (0)211 81-15230

Si E-MAIL:

keller@phil-fak.uni-duesseldorf.de

SI INTERNET:

www.phil-fak.uni-duesseldorf.de/rudi.keller 